\title{
Impacto de la Ley 14/2007 de Investigación Biomédica en los ensayos clínicos
}

\author{
Miguel Ángel Ramiro Avilés \\ Área de Filosofía del Derecho. Universidad Carlos III. Instituto de Derechos Humanos Bartolomé de las Casas. \\ Comité Ético de Investigación Clínica del Hospital Universitario de Getafe. Madrid. España.
}

El objetivo del artículo es exponer de qué manera afecta la Ley 14/2007, de Investigación Biomédica, a la realización de ensayos clínicos con medicamentos en España, mostrando las luces y las sombras que proyecta dicha norma jurídica.

\begin{abstract}
¿Qué entiende la Ley 14/2007 por investigación biomédica?
La entrada en vigor de la Ley 14/2007, de Investigación Biomédica ${ }^{1}$, determina que toda la investigación biomédica que se realice en España deba cumplir con sus preceptos. A la vista de esta norma, la primera cuestión es saber qué se entiende por investigación biomédica. La respuesta está en el artículo 1.1 cuando establece su objeto y alcance enumerando una serie de actividades, tales como: a) las investigaciones relacionadas con la salud humana que impliquen procedimientos invasivos; b) la donación y utilización de ovocitos, espermatozoides, preembriones, embriones y fetos humanos o de sus células, tejidos u órganos con fines de investigación y sus posibles aplicaciones clínicas; c) el tratamiento de aplicaciones biológicas; d) el almacenamiento y movimiento de muestras biológicas, y e) los biobancos. El artículo 1.3 determina negativamente el objeto y alcance, pues excluye de forma expresa los ensayos clínicos con medicamentos y productos sanitarios, y remite su regulación a la legislación especial.
\end{abstract}

\section{Los ensayos clínicos con medicamentos en la Ley 14/2007}

A tenor del objeto y ámbito de aplicación de la Ley 14/2007, una primera conclusión podría ser que dicha ley no afecta a los ensayos clínicos con medicamentos, ya que no se derogan ni los artículos que se dedican a los ensayos clínicos con medicamentos en la Ley 29/2006, de 26 de julio, de Garantías y Uso Racional de los Medicamentos y Productos Sanitarios, ni el Real Decreto (RD) 223/2004, de 6 de febrero, de Ensayos Clínicos con Medicamentos². La Ley 14/2007 vendría, más bien, a reforzar la vigencia de este último en cuanto norma especial (1). Esa primera conclusión es errónea, ya que la Ley 14/2007 sí afecta a los ensayos clínicos con medicamentos, pues forman parte de la investigación biomédica si se tiene en cuenta la definición de «procedimiento invasivo»: una intervención en seres humanos que se realiza con fines de investigación e implica un riesgo físico o psíquico para el sujeto afectado (art. 3.t de la Ley 14/2007). Los ensayos clínicos con medicamentos son

Correspondencia: Prof. Dr. M.A. Ramiro Avilés.

Instituto de Derechos Humanos Bartolomé de las Casas. Universidad Carlos III. Madrid, 126. 28903 Getafe. Madrid. España.

Correo electrónico: miguelangel.ramiro@uc3m.es

Recibido el 26-10-2007; aceptado para su publicación el 2-1-2008. un tipo de investigación biomédica que supone una intervención sanitaria en seres humanos mediante la evaluación experimental de una sustancia o medicamento no autorizado como especialidad farmacéutica o en condiciones de uso distintas de las autorizadas, y que busca poner de manifiesto sus efectos farmacodinámicos; recoger datos referentes a su absorción, distribución, metabolismo y expulsión en el organismo humano; establecer su eficacia para una indicación terapéutica, profiláctica o diagnóstica determinada; conocer el perfil de sus reacciones adversas, y establecer su seguridad. Como en toda experimentación, los sujetos que participan están expuestos a una serie de daños e inconvenientes que pueden producirse en su salud física o psíquica como consecuencia de dicha evaluación experimental, pero en ningún caso dichos daños e inconvenientes pueden ser desorbitados, pues se han ponderado con respecto a los beneficios previsibles. El ensayo debe estar diseñado para reducir al mínimo el posible dolor y siempre debe prevalecer el bienestar del sujeto sobre el interés de la ciencia o de la sociedad.

Este tipo de investigación biomédica se realiza para mejorar tanto la calidad como la esperanza de vida y el bienestar de las personas reduciendo las tasas de mortalidad y morbilidad de las enfermedades que pueden afectar a su salud. El fomento de este tipo de investigación se enmarca dentro de las obligaciones positivas que en forma de políticas públicas las Administraciones estatal y autonómicas deben ejecutar para lograr la efectiva realización del derecho a la protección de la salud reconocido en el art. 43 de la Constitución española. Además, en nuestro sistema jurídico se ha consagrado el derecho de los pacientes a ser advertidos de la posibilidad de utilizar los procedimientos de pronóstico, diagnóstico y terapéuticos que se apliquen en un proyecto docente o de investigación (art. 8.4 de la Ley 41/2002, de 14 de noviembre, Básica Reguladora de la Autonomía del Paciente y de Derechos y Obligaciones en Materia de Información y Documentación Clínica ${ }^{3}$ ). La Ley 14/2007 se enmarca en la tarea de construcción de un marco normativo adecuado para que la investigación biomédica pueda realizarse en España con todas las garantías protegiendo los derechos de las personas que participen en ella. Recoge los principios y criterios, de cada vez más amplia aceptación, a

(1) El artículo 2.a del RD 223/2004 señala que un ensayo clínico con medicamentos es «toda investigación efectuada en seres humanos para determinar o confirmar los efectos clínicos, farmacológicos y/o demás efectos farmacodinámicos, y/o de detectar las reacciones adversas, y/o de estudiar la absorción, distribución, metabolismo y excreción de uno o varios medicamentos en investigación con el fin de determinar su seguridad y/o su eficamentos en inver cia». Por otro lado, un medicamento en investigación ses toda "forma farmacéutica de una sustancia activa o placebo que se investiga o se utiliza como referencia en un ensayo clínico, incluidos los productos con autorización de comercialización cuando se utilicen o combinen (en la formulación o en el envase) de forma diferente de la autorizada, o cuando se utilicen para tratar una indicación no autorizada, o para obtener más información sobre un uso autorizado» (art. 2.d del RD 223/2004). 
partir de los cuales se han construido las normas y reglas de conducta con las que se debe lograr el establecimiento de un necesario equilibrio entre las necesidades de los investigadores y la confianza de la sociedad en la investigación científica. En ese sentido, tiene como uno de sus ejes prioritarios asegurar el respeto y la protección de los derechos fundamentales y las libertades públicas del ser humano y de otros bienes jurídicos relacionados con ellos. Así, se reconoce que la Ley 14/2007 se ha construido «sobre los principios de la integridad de las personas y la protección de la dignidad e identidad del ser humano en cualquier investigación biomédica que implique intervenciones sobre seres humanos, así como en la realización de análisis genéticos, el tratamiento de datos genéticos de carácter personal y de las muestras biológicas de origen humano que se utilicen en investigación».

Por todo ello, desde que en 1975 la Asociación Médica Mundial revisase en Tokio la Declaración de Helsinki, de Principios Éticos para las Invesigaciones Médicas en Seres Humanos, la autorización de cualquier tipo de investigación biomédica debe ser previamente evaluada por un comité independiente, que en España, a raíz de la Ley 14/2007, se denomina «comité de ética de la investigación»(CEI). Es aquí precisamente donde comienzan los problemas, porque hasta la entrada en vigor de la Ley 14/2007 los ensayos clínicos con medicamentos tenían que contar con un informe previo favorable de un CEI clínica (CEIC), por ser el órgano que estaba encargado de «velar por la protección de los derechos, seguridad y bienestar de los sujetos que participen en un ensayo y de ofrecer garantía pública al respecto, mediante un dictamen sobre el protocolo del ensayo, la idoneidad de los investigadores y la adecuación de las instalaciones, así como los métodos y los documentos que vayan a utilizarse para informar a los sujetos del ensayo con el fin de obtener su consentimiento informado» (art. 2.n del RD 223/2004)

A pesar de que la Ley 14/2007 expresamente remite a la legislación especial, no es posible obviar la Disposición Transitoria Tercera, donde se señala que «los Comités Éticos de Investigación Clínica dejarán de existir a partir del momento en que se constituyan los Comités de Ética de la Investigación. Hasta que dichos Comités se constituyan, los Comités Éticos de Investigación Clínica que estén en funcionamiento en los centros que realicen investigación biomédica podrán asumir las competencias de aquéllos». Podría pensarse que esta afectación no es en absoluto grave, ya que las personas que participen en un ensayo clínico con medicamentos siguen contando con la garantía de un órgano de control de la calidad metodológica, ética y legal de la investigación. Si bien lo anterior es cierto, es necesario que la gravedad del asunto se observe no tanto desde el fondo como desde la forma; esto es, no tanto desde la óptica de los derechos de las personas que participan en un ensayo clínico con medicamentos, como desde la óptica de cómo ha sido el procedimiento de sustitución del CEIC por el CEl.

Una de las novedades que introduce la Ley 14/2007 es la creación de los CEI y del Comité de Bioética de España (arts. 12 y 77). Dejando a un lado la oportunidad que ha supuesto la creación del Comité de Bioética de España como órgano consultivo para que las implicaciones éticas y sociales de la biomedicina y las ciencias de la salud se debatan sensatamente y serenamente, el procedimiento diseñado para que Ios CEI sustituyan a los CEIC no ha sido el más oportuno y deja bastante que desear desde el punto de vista de cómo debe actuar la Administración en un Estado de derecho.

Por lo que respecta a la definición de qué es un CEI y cuáles son sus funciones, se señala que los CEl evaluarán la cualificación del investigador principal y la del equipo investigador, así como la factibilidad de los proyectos de investigación; ponderarán los aspectos metodológicos, éticos y legales de los proyectos; ponderarán el balance de riesgos y beneficios anticipados dimanantes; velarán por el cumplimiento de procedimientos que permitan asegurar la trazabilidad de las muestras de origen humano; emitirán un informe, previa evaluación del proyecto, de toda investigación biomédica que implique intervenciones en seres humanos o utilización de muestras biológicas de origen humano (art. 12 de la Ley 14/2007). Pues bien, a pesar de que la Ley 14/2007 anula la existencia de los CEIC, no contempla expresamente entre las funciones de los CEI la evaluación de los ensayos clínicos con medicamentos, lo que hubiera sido aconsejable dada la reconocida especificidad al remitirlos a su legislación especial, y se limita a señalar de forma genérica que toda investigación biomédica que implique intervenciones en seres humanos, entre las que se incluyen los ensayos clínicos con medicamentos, deberá contar con el informe favorable del CEI. A pesar de lo anterior, como es obvio, una vez constituidos, los CEI deberán asumir las competencias de los extintos CEIC y aplicar el RD 223/2004 cuando se trate de la evaluación de un ensayo clínico con medicamentos en seres humanos.

Pero los problemas no terminan aquí, porque en la Ley $14 / 2007$ no viene fijado el momento temporal en que deben estar constituidos los CEI. No existe un plazo perentorio que deban cumplir los centros que realicen investigación biomédica. No obstante, los centros de investigación no pueden eludir el cumplimiento de la norma si quieren seguir realizando investigación biomédica que no sea un ensayo clínico con medicamentos, porque para ello deberán contar con un CEI que emita un informe favorable del desarrollo de cualquier proyecto de investigación sobre seres humanos o su material biológico (art. 2.e de la Ley 14/2007). En este apartado la regulación legal también es deficiente, ya que hubiera sido aconsejable que se fijase un plazo máximo en el que los CEIC deberían convertirse en CEI.

En cuanto a la formación del CEI, la Ley 14/2007 señala que la composición interdisciplinaria será uno de los criterios que se tendrán en cuenta a la hora de acreditarse ante el órgano competente de la comunidad autónoma que corresponda. A diferencia de lo que ocurría hasta ahora, no se señala expresamente la necesidad de contar con profesionales concretos como farmacólogos clínicos, representantes del servicio de farmacia del hospital, diplomados universitarios en enfermería o licenciados en derecho (art. 12 del RD 223/2004). Tampoco se hace referencia a lo importante e interesante que sería que el CEI contara con personas con formación en bioética, como ocurre en los comités de ética para la asistencia sanitaria, o que se pudiera contar con la colaboración de expertos externos cuando los miembros del CEI no reúnan los conocimientos y experiencia necesarios para evaluar un determinado proyecto de investigación biomédica. En este sentido, el artículo 14.4 del RD 223/2004 es sumamente clarificador: "Cuando el Comité Ético de Investigación Clínica no reúna los conocimientos y experiencia necesarios para evaluar un determinado ensayo clínico recabará el asesoramiento de alguna persona experta no perteneciente al comité, que respetará el principio de confidencialidad. De esta manera: a) cuando el comité evalúe protocolos de investigación clínica con procedimientos quirúrgicos, técnicas diagnósticas o productos sanitarios, contará con el asesoramiento de al menos una persona experta en el procedimiento o tecnología que se vaya a evaluar y $b$ ) Cuando el comité evalúe ensayos clínicos que se refieran a menores o a sujetos incapacitados, contará con el asesora- 
miento de al menos una persona con experiencia en el tratamiento de la población que se incluya en el ensayo». A pesar del silencio, si se quiere cumplir con la función de "ponderar los aspectos metodológicos, éticos y legales del proyecto de investigación», la formación del CEI deberá ser multidisciplinaria, y es conveniente tener en cuenta que no se veda la posibilidad de que los CEIC se transformen automáticamente en $\mathrm{CEI}$, manteniendo incluso a todos sus miembros, aunque, dada la ampliación de las competencias que tiene el CEl, sería conveniente contar con nuevos miembros que aumentasen la multidisciplinariedad.

La Ley 14/2007 también tiene algunos problemas de redacción que afectan a la depurada técnica legislativa que se espera de una norma con rango de ley. En este sentido, en el apartado IV de la «Exposición de motivos» se señala que se crean los "Comités de Investigación Biomédica», pero en el articulado no aparecen dichos comités. Suponemos que se está haciendo referencia a los CEI. Otro ejemplo en el que la redacción debería haber estado más cuidada es cuando en el artículo 4.5 se hace referencia a un "comité asistencial». ¿A qué comité asistencial se refiere? Suponemos que es el comité de ética para la asistencia sanitaria, ya que el texto admite la posibilidad de que dicho comité asistencial no exista en el centro en el que vaya a realizarse la investigación biomédica. Por último, esta ley debería haber tenido en cuenta qué va a pasar con el centro coordinador de los CEIC (art. 9 del RD 223/2004). Esto es importante porque la Ley 14/2007 copia la experiencia del RD 223/2004 en lo relativo al dictamen único, pero olvida los mecanismos de coordinación que se requieren. La principal pregunta que debería contestarse es si dicho centro pasaría a coordinar los CEI tanto cuando evalúen investigación biomédica que no constituye un ensayo clínico con medicamentos como cuando evalúen ensayos clínicos con medicamentos, básicamente con la finalidad de facilitar el dictamen único en los ensayos multicéntricos.

\section{Algunas sombras de la Ley 14/2007 respecto a otros tipos de investigación biomédica}

Si se fija la atención sobre la finalidad general de la Ley 14/2007 de asegurar el respeto y la protección de los derechos de las personas que participan en cualquier investigación biomédica que implique una intervención sobre ellas, la norma adolece de varios defectos que pueden impedir que cumpla con su objetivo. Cabe destacar, en primer lugar, la cuestión de la remuneración de las personas sanas o enfermas que participan en la investigación biomédica que implica procedimientos invasivos y no son ensayos clínicos con medicamentos. El hecho de que por razones biológicas la investigación biomédica, en última instancia, tenga que realizarse en seres humanos plantea problemas éticos cuando se contempla en el proyecto la posibilidad de que las personas que participen en un ensayo reciban una remuneración económica. En este sentido, en las normas especiales que regulan los ensayos clínicos con medicamentos se establece que, cuando el ensayo clínico con medicamentos no tiene un beneficio potencial directo para las personas que participan, éstas podrán recibir una compensación monetaria o en especie, previamente pactada, por las molestias sufridas (art. 3.8 del RD 223/2004). Dicha compensación en ningún caso podrá ser tan elevada como para inducir a una persona a participar en el ensayo clínico por motivos distintos del interés por el avance de la ciencia. Este supuesto es bastante normal cuando estamos ante un ensayo clínico con medicamentos en fase 1, pues sólo en esa fase se admite la participación de personas sanas. Di- cha participación es necesaria y se justifica porque proporciona información preliminar sobre el efecto y la seguridad del medicamento que orientará la pauta de administración más apropiada para fases posteriores. En todo caso, el CEIC evalúa la oportunidad de la compensación y su cuantía, y se determina su idoneidad o falta de idoneidad, si puede inducir a una persona a participar en un ensayo clínico por razones diferentes del avance de la ciencia. Cuando se trata de personas que pueden tener un beneficio potencial directo, el artículo 3.9 del RD 223/2004 prohíbe la remuneración al señalar que sólo podrán recibir el reintegro de los gastos extraordinarios y pérdidas de productividad que se deriven de su participación en el ensayo. Por dicho motivo, en muchos protocolos de ensayos clínicos con medicamentos se informa a los posibles candidatos a participar de que sólo se les reintegrarán los gastos extras que puedan justificarse debidamente mediante una factura o un recibo, lo cual entra en la lógica de gratuidad que está presente en la realización de un ensayo. Por último, en el artículo 3.8 del RD 223/2004 se dice que no podrá haber ningún tipo de compensación económica, a excepción del reintegro de los gastos extraordinarios y pérdidas de productividad, cuando las personas que participan en un ensayo clínico con medicamentos sin beneficio potencial directo sean menores de edad o personas incapaces, con el fin de evitar su posible explotación. Pues bien, a pesar de que existía este precedente legislativo, la Ley 14/2007 deja esta cuestión en el aire, ya que no señala nada concreto al respecto. La norma, en su artículo 18 , se limita a señalar las obvias compensaciones que las personas pueden recibir por los daños sufridos como consecuencia de su participación en un proyecto de investigación. La Ley 14/2007 no resuelve la duda de si se puede o no remunerar a las personas que participan en una investigación que implica un procedimiento invasivo, pero que no constituye un ensayo clínico con medicamentos. Entiendo que, si este caso no se prohíbe expresamente, la remuneración está permitida y que será el CEl el que, en su caso, deberá establecer si la cuantía fijada es aceptable o no. Posiblemente ése sea el caso más fácil de resolver. No sería tan sencillo saber si el CEI podría, por ejemplo, rechazar o plantear objeciones de carácter ético cuando una investigación que implique un procedimiento invasivo, no constituya un ensayo clínico con medicamentos y tenga un posible beneficio directo para la persona que interviene, prevea una compensación económica. ¿Y si la remuneración se establece en una investigación en que intervienen personas menores de edad o con una discapacidad temporal o permanente que les impide decidir libremente? Imagínese por un momento, sin olvidar la singularidad que tienen los CEI como órganos administrativos porque sus decisiones son vinculantes, que un CEI deniega la autorización para realizar una investigación que implica un procedimiento invasivo, pero no constituye un ensayo clínico con medicamentos, porque establece una compensación económica para una persona mayor de edad que potencialmente podría beneficiarse, o cuando intervienen personas menores de edad o con una discapacidad temporal o permanente que les impide decidir libremente, ¿podría el investigador principal o el promotor de la investigación plantear un recurso administrativo ordinario por considerar el interesado que la resolución afecta a la libertad de investigación reconocida en el artículo 20.1.b de la Constitución? En mi opinión, en los casos en que la Administración pueda afectar a alguno de los derechos y libertades reconocidos en la Constitución, es recomendable que las causas que permiten dicha afectación estén tasadas legalmente con el fin de aumentar la certeza en la aplicación del derecho, ya que, a la vista de la experiencia de los 
CEIC, la apelación a los principios éticos puede resultar insuficiente e injusta desde el punto de vista de la aplicación de las normas.

De igual forma, es flagrante la parquedad con que la Ley 14/2007 trata las situaciones específicas que supone la investigación en personas que no tengan capacidad para expresar su consentimiento y la investigación en personas incapaces de consentir debido a su situación clínica (arts. 20 y 21). Respecto a los menores de edad, englobados junto a las personas incapacitadas por una resolución judicial, cabe señalar en primer lugar que, aunque en el ámbito sanitario la mayoría de edad se obtiene a los 16 años, dicha regla no opera cuando se trata de la participación en ensayos clínicos con medicamentos. En esos casos, la mayoría de edad se sigue obteniendo a los 18 años, por lo que serán los padres, tutores o representantes legales quienes reciban la información y consientan en la participación en el ensayo clínico (art. 9.3.c de la Ley 41/2002).

La forma en que la Ley 14/2007 trata la información y el consentimiento de los menores de edad y de las personas con discapacidad en la investigación biomédica que supone una intervención invasiva, pero no es un ensayo clínico con medicamentos, es deficiente, porque simplemente señala que la persona que vaya a participar debe haber sido informada por escrito de sus derechos y de los límites prescritos en la propia Ley de Investigación Biomédica y en la normativa que la desarrolle, y excepciona ese derecho de información cuando se trate de personas que por sus circunstancias no estén en situación de recibir información (art. 20.1.c). Parece que la Ley $14 / 2007$ olvida que no todos los menores de edad y no todas las personas con discapacidad son iguales, y que dependiendo de su edad y del tipo de discapacidad pueden ser capaces de comprender la información clínica siempre y cuando se adapte a su nivel de entendimiento. Por otro lado, además de negarles una información adecuada, les niega prácticamente toda capacidad de oponerse a su inclusión en una investigación biomédica, ya que son únicamente los representantes legales quienes tienen que consentir y sólo a ellos va dirigida la obligación de tener en cuenta los deseos u objeciones previamente expresados por la persona afectada (art. 20.1.d). Esto último es perfectamente lógico, ya que quien no está informado de forma adecuada no puede consentir.

Pues bien, dicha regulación olvida el avance que supuso el RD 223/2004 en el tratamiento de la participación en ensayos clínicos con medicamentos tanto de menores de edad como de personas con discapacidad, ya que no sólo se garantiza su derecho a acceder a una información adaptada a su nivel de entendimiento, lo cual en muchos casos exige que haya hojas de información especiales, sino que forman parte activa del proceso de consentimiento y, además, la obligación de tener en cuenta su oposición a participar en el ensayo clínico con medicamentos se extiende a los investigadores. En este sentido, si el sujeto del ensayo clínico con medicamentos tiene 12 años o más, deberá prestar su consentimiento para participar en el ensayo y a tal efecto recibirá, por parte de personal que cuente con experiencia en el trato con menores, una información sobre el ensayo, los riesgos y los beneficios que sea adecuada a su capacidad de entendimiento. El investigador, además, aceptará el de- seo explícito del menor de negarse a participar en el ensayo o de retirarse en cualquier momento, cuando éste sea capaz de formarse una opinión en función de la información recibida. Por tal motivo, la participación de menores con 12 años o más depende tanto de la voluntad de los padres como del menor (art. 7.3.a del RD 223/2004). Por su parte, las personas adultas sin capacidad de otorgar su consentimiento de forma temporal o permanente, cuando sus condiciones lo permitan, deberán prestar su consentimiento y para ello recibirán información adaptada a su nivel de entendimiento. El investigador, por último, deberá tener en cuenta la voluntad de retirarse del ensayo que exprese la persona adulta sin capacidad de otorgar su consentimiento de forma temporal o permanente (art. 7.3.b del RD 223/2004).

Por mor de las garantías que deben exigirse en este tipo de actuaciones, no parece razonable que en la Ley 14/2007 sólo se exija que el Ministerio Fiscal esté informado cuando la investigación en menores de edad y en personas con discapacidad no vaya a producir resultados en beneficio directo para la salud de esas personas (art. 20.2.c). De nuevo el RD 223/2004 parece más razonable cuando señala que el promotor de un ensayo clínico tiene que poner en conocimiento del Ministerio Fiscal las autorizaciones de los ensayos clínicos cuya población incluya a menores, indistintamente si va o no va a producir resultados en beneficio directo para la salud de esas personas.

En el caso de las mujeres embarazadas o en período de lactancia sí se ha sido más cuidadoso, ya que predomina el espíritu garantista tanto de la mujer como de los embriones, fetos o niños cuando la investigación no tiene un potencial beneficio directo. Así, se podrá autorizar una investigación de este tipo en que participe una mujer embarazada o en período de lactancia exclusivamente en las siguientes condiciones: que la investigación tenga como finalidad obtener un conocimiento que redunde en beneficio de otras mujeres, embriones, fetos o niños; que no pueda realizarse una investigación de eficacia comparable en mujeres que no estén embarazadas o en período de lactancia; que entrañe unos riesgos y perjuicios mínimos para la mujer, el embrión, el feto o el niño; que la mujer embarazada o los representantes legales del niño presten su consentimiento (art. 19 de la Ley 14/2007). En este punto sólo cabe subrayar la necesidad de que, cuando se informe a la mujer embarazada de los riesgos y perjuicios que se pueden ocasionar al embrión o al feto, se insista en que en caso de producirse un daño tendría derecho a solicitar la interrupción voluntaria del embarazo si puede considerarse que dicho daño, según la opinión de 2 especialistas, ha causado al feto una grave malformación física y/o psíquica.

\section{REFERENCIAS BIBLIOGRÁFICAS}

1. Ley 14/2007, de 3 de julio, de Investigación Biomédica. BOE n. ${ }^{\circ} 159$, de 4 de julio de 2007. p. 28826-48.

2. Real Decreto $223 / 2004$, de 6 de febrero, por el que se regulan los ensayos clínicos con medicamentos. BOE n. ${ }^{\circ} 33$, de 7 de febrero de 2004. p. 5429-43.

3. Ley $41 / 2002$, de 14 de noviembre, Básica Reguladora de la Autonomía del Paciente y de Derechos y Obligaciones en Materia de Información y Documentación Clínica. BOE n. ${ }^{\circ} 274$, de 15 de noviembre de 2002. p. 40126-32. 